\title{
In-situ Observations of a Multi-Asperity Real Contact Area on a Submicron Scale
}

\author{
Blaž Brodnik Žugelj - Mitjan Kalin* \\ University of Ljubljana, Faculty of Mechanical Engineering, Slovenia
}

We present apparatus that allows in-situ optical measurements of the evolving real contact area between a rigid glass and a deformable Al6026 surface with $700 \mathrm{~nm}$ of lateral and $20 \mathrm{~nm}$ of vertical resolution. In previous experimental studies of multi-asperity real contact area this was investigated either with much less accuracy or did not include the full (loaded) nominal contact area, which can hinder the relevant sub-micron deformation phenomena. During experiments involving the real contact area, the contact load and asperity deformations are simultaneously measured. To show the relevance of the developed experimental procedure measurements are compared to the results calculated with the Greenwood-Williamson (GW) and a modified Abbott-Firestone $(A F(H))$ models, which represent the two extreme deformation-regime models. The $A F(H)$ model shows relatively good agreement between the real contact area and the asperity deformations ( $<60 \%)$, while the GW model deviates by up to 10 times, depending on the deformation value. In contrast, the GW model shows better agreement for the relationship between the contact load and the asperity deformation (<20\%), while the $A F(H)$ deviates by more, approximately $30 \%$. The results also indicate that the real contact area is a non-linear function of the contact load, while theoretical models predict their linearity. Finally, it is demonstrated that the real contact area reaches only up to $9 \%$ of the nominal value in the loading range up to the material yield strength, as calculated for the nominal contact parameters.

Keywords: test rig, in-situ experiment, optical technique, asperities, real contact area

Highlights

- A test rig for in-situ experimental investigations of a multi-asperity contact under static loading conditions is presented.

- The contact between a deformable flat Al6026 specimen and a transparent rigid flat is investigated.

- The contact load, the asperity deformations in vertical direction and Ar/An are analyzed.

- $\quad$ Theoretical results of Greenwood-Williamson model and modified Abbott-Firestone model are compared to the experimental results.

- The comparison between the theoretical and experimental results is discussed.

\section{INTRODUCTION}

In a conventional engineering approach the contact conditions are normally calculated with the assumption of a nominal contact area between two surfaces, which, under certain conditions, can significantly over-estimate the size of the actual contact area. A consideration of such contact conditions leads to calculations of milder contact conditions than exist in reality.

The problem of the real contact area has been investigated using several different approaches, where theoretical investigations tend to predominate. Probably the best-known real-contact-area model is the Greenwood-Williamson (GW) model from 1966 [1]. Because of several general assumptions, on which the GW model is based, a number of modifications to the model have appeared. Some of them show improvements in terms of mechanical characterization [2] to [5], while numerous studies have been made on geometrical treatments [6] to [8]. Based on investigations of the real contact area it is obvious that the problem is complex and involves many aspects.
One of the most important issues with a theoretical investigation of the real contact area is the geometry of the engineering surfaces and their behaviour when in contact. Based on geometric characterization of contacting surfaces contact models can be divided into four groups, i.e., statistical [9] to [11], fractal [12] and [13], multi-scale [14] to [16] and deterministic [17] to [19]. While statistical models are the most widely used because of their simple adoption and good approximation to actual engineering surfaces, they are not a very powerful tool for a detailed contact analysis since the surface properties are determined with statistical functions. For a more detailed geometrical characterization of the surfaces, different measuring tools (contact profilometer, atomic-force microscope (AFM), scanning electron microscope (SEM), optical interferometer, etc.) can be used. The question here is the resolution of the different techniques, because the same surface can be characterized differently when different techniques are used. To overcome this problem, i.e., a non-uniform surface characterization, fractal methods of surface characterization can be adopted. The main advantage of fractal methods lies in a uniform surface characterization that is independent 
of the measuring technique. On the other hand, some problems are also connected to fractal contact theory. Namely, the distribution of contact areas is assumed geometrically without a consideration of the actual elasticity and it predicts that lighter loads cause greater percentage of plastically deformed contacts. The shortcomings of fractal models are addressed in multiscale models, which similar as fractal models consider multi-scale effects of the surface but incorporate more accurate deformation mechanics [14] and [16].

Another type of geometric characterization of contacting surfaces presents deterministic method [20]. With this method, first, the real engineering surface is measured with one of the surface-measuring techniques. Then, in the next step, different criteria for identifying asperity peaks can be applied [21]. According to the implemented criterion, the number of detected asperity peaks, their locations and the individual asperity-tip radii can be accurately determined. Consequently, a surface topography that is the subject of the contact analysis is set based on actual surface measurements, which makes the analysis more accurate than in the case of using statistical methods. In any case, even the deterministic approach has some limitations. The main shortcoming of the deterministic approach is related to the application of the correct criterion for identifying the asperities. Thus far, we do not know which criterion is the most accurate for the appropriate detection and characterization of asperities [21].

Every theoretical model for determining a real contact area has some advantages and disadvantages. A common disadvantage of all theoretical approaches is simplification, which relates either to assumptions about asperity deformations or their geometrical properties. For an accurate investigation of the real contact area and understanding the actual contact, an experimental approach must be adopted that makes it possible to facilitate theoretical approaches with the actual measurements. In the literature [22] to [31] several experimental techniques are described for real-contact-area measurements. Probably the most practical in-situ methods for real-contact-area measurements are based on the electrical [24] and thermal [25] contact resistance, as well as ultrasonic [26] and [27] and optical [28] and [29] principles. However, the most common technique for a realcontact-area measurement is the optical method, the only critical limitation of which is the transparency of one of the contacting bodies so the light can pass through into the contact. The main advantage of optical methods is that the asperity junction can be "seen", which provides trust and an intuitive understanding of the physical processes occurring. Several attempts to experimentally analyse a real contact area with an optical method have been reported [32] to [36]. The vast majority of research has involved the contact between an ideally smooth, rigid flat and a ball, which simulates a single asperity peak [29], [35], [37] and [38].

Multi-asperity contacts and the real contact area between two realistic and rough flat surfaces have rarely been investigated experimentally [23], [28] and [30], and no detailed study with a submicron resolution of asperity growth has been presented, to the best of our knowledge. However, multi-asperity contact analyses are of great importance since the asperities interact with each other and influence how much load each asperity will carry. This means that their simultaneous interaction and growth should be understood for the proper development of a realcontact-area model.

In this work an experimental investigation of the real contact area between "ideally" smooth and rough, nominally flat surfaces with an in-situ optical technique on a submicron scale is presented. The experimentally obtained results are compared with theoretical predictions, which are calculated with two fundamental, commonly applied contact models with the two extreme deformation situations; fully elastic and fully plastic. GW model is valid for solely elastically deformed surfaces and the Abbott-Firestone (AF) model, modified with hardness criteria (see Experimental section) $\mathrm{AF}(H)$, assumes only plastic deformation of the contacting surfaces. Namely, the main motivation for the present investigation is not to evaluate how accurate these two contact models predict the actual behaviour, but to compare actual contact behaviour with the two possible extreme situations of the contact-deformation regimes. In fact, it is well-known and expected that the actual contact behaviour is elastic-plastic, however, several elastic-plastic models, which all have many specifics and details require in-depth comments and analyses when presented, which greatly exceeds the scope of this work. A detailed comparison and evaluation of different elastic-plastic models with the same experimental technique is addressed in [39].

\section{EXPERIMENTAL}

\subsection{Test Apparatus}

A test apparatus was designed for in-situ measurements of the real contact area between two surfaces on the submicron scale (Fig. 1). The set-up 
allows normal loading of a deformable, multi-asperity, nominally flat specimen against a transparent rigid window where the changes to the contact area are captured by an optical microscope, simultaneously with the deformation in the vertical direction and the contact load.

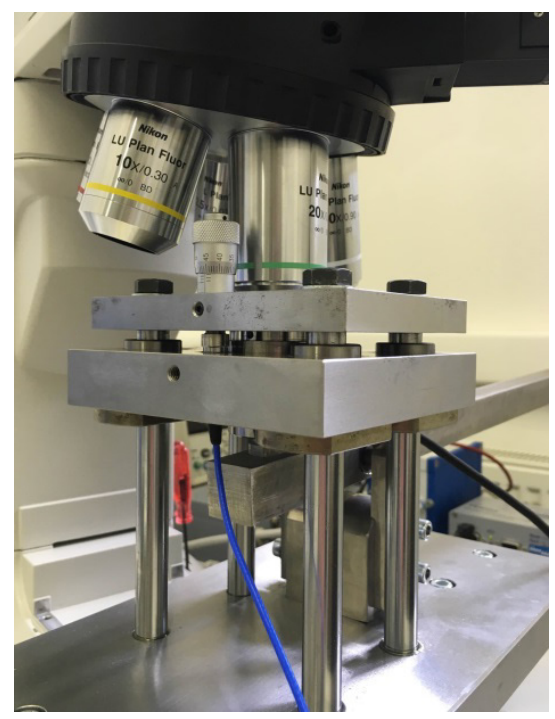

Fig. 1. Test apparatus for in-situ measurements of the real contact area

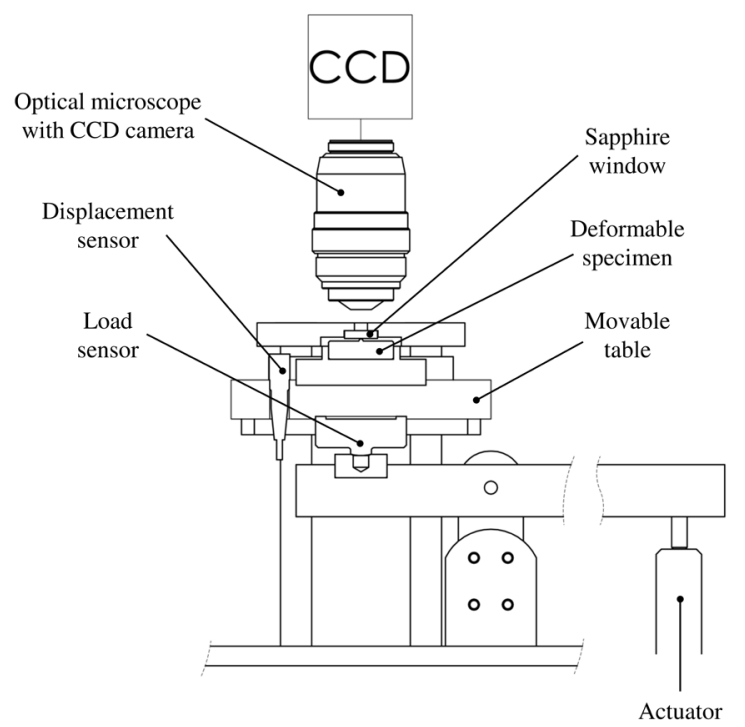

Fig. 2. Schematic presentation of the test rig that was developed for investigating the real contact area between the transparent rigid window and deformable, multi-asperity, nominally flat specimen

Fig. 2 shows a schematic of the test apparatus, which consists of the following components: a whitelight optical microscope with a charge-coupled device (CCD) camera; a rigid, "ideally" smooth, flat sapphire window; a deformable specimen; a displacement sensor, a table that is movable in the z-direction; and an actuator. A very accurate actuator is able to press the specimen against the transparent sapphire window at a constant velocity as low as $10 \mathrm{~nm} / \mathrm{s}$. The contact load is detected with a resolution of $0.1 \mathrm{~N}$ using a compressive-force transducer (AEP transducers, Italy), which is installed between a lever and the movable table. An accurate capacitive displacement sensor (Micro-Epsilon, Germany) with a resolution of $20 \mathrm{~nm}$ measures the deformation of the specimen as it is pressed against a rigid flat. In order to magnify the contact between the deformable specimen and the transparent sapphire window, high-resolution $(2,560 \times 1,920$ pixels $)$ images are captured with an optical microscope (Eclipse LV 150, Nikon, Japan) using 200× magnification and equipped with a CCD camera (DS-fi1, Nikon, Japan) using a frame rate of $1 \mathrm{~Hz}$ and a lateral resolution of $700 \mathrm{~nm}$. The output signals, detected by load and displacement sensors, are captured with a data-acquisition card. Commercial software (Labview 2013, National Instruments, USA) is used for the signal processing, full control of the actuator and synchronised storage of the measured displacement, the contact load and the captured images.

\subsection{Specimens and Methods}

A commercial 6026 aluminium alloy was used in this investigation (Table 1). Young's modulus $(E)$, Poisson's ratio $(v)$ and yield strength $(Y)$ were provided by the manufacturer while we measured Vickers hardness (HV) with a Leitz Miniload microhardness tester (Leitz Miniload, Wild Leitz GmbH, Germany). The measured dimensionless Vickers hardness was converted in $\mathrm{MPa}$ so it could be used in theoretical analysis (see Section 1.3).

First, the specimens were cut out from a rod with a circular profile. The roughness, which in this study was Ra $0.6 \mu \mathrm{m}$, was obtained with a sequence of grinding and polishing steps using a surface-grinding machine (RotoPol-21 with RotoForce-3 module, Struers, Denmark). The surface-roughness parameters were measured using a stylus-tip profilometer (T8000, Hommelwerke GmbH, Germany) with a TKE100/17 probe $\left(90^{\circ}\right.$ angle and $5 \mu \mathrm{m}$ radius) according to the DIN 4768 standard at cut-off length $0.8 \mathrm{~mm}$ and traversing length $4.8 \mathrm{~mm}$. After the surfaces were prepared, the samples were carefully milled to form a cone shape with a circular flat top and a pre-prepared roughness. The diameter of the remaining flat surfaces was approximately $200 \mu \mathrm{m}$ (Fig. 3), resulting in 
around $0.03 \mathrm{~mm}^{2}$ of nominal contact area $(A n)$. It is especially important to note that under all the testing conditions in this study, the whole nominal contact area was captured in the objective view. Consequently, all the asperities were detected when in contact. Prior to the experiment, each specimen was cleaned with acetone and ethanol so as to remove any contaminants from the surface.

Table 1. Mechanical properties of the tested aluminium 6026 specimen and the sapphire window

\begin{tabular}{lcc}
\hline Material & Aluminium 6026 & Sapphire \\
\hline Young's modulus $(E),[\mathrm{MPa}]$ & 69,000 & 335,000 \\
\hline Poisson's ratio $(v),[/]$ & 0.33 & 0.25 \\
\hline Hardness $(H),[\mathrm{MPa}]$ & 1,370 & 27,130 \\
\hline Yield strength $(Y),[\mathrm{MPa}]$ & 320 & $\mathrm{~N} / \mathrm{A}$ \\
\hline
\end{tabular}
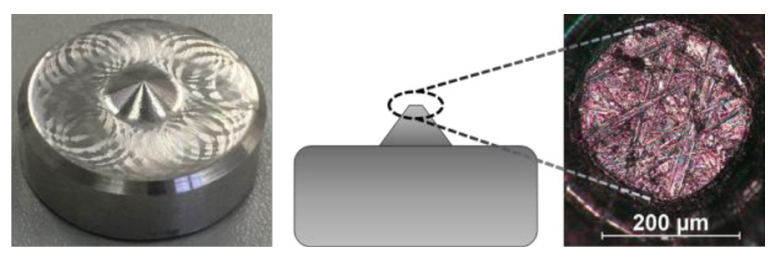

Fig. 3. Final shape of the tested aluminium specimens and a detailed view of the top surface

Each experiment began by mounting the specimen on a movable table. In the next step the actuator slowly lifted the table with the specimen into contact with the rigid flat at a constant velocity of 50 $\mathrm{nm} / \mathrm{s}$. While the sample was being pressed against the sapphire window, the displacement and load sensors were measuring the vertical deformation of the sample and the contact load, respectively. For the purposes of this investigation the experiments were controlled by the contact load. The actuator pressed the specimen against the sapphire window until a preset contact load was achieved. The maximum contact load was limited to the value where the resulting nominal contact pressure is equal to the yield strength of the material $(Y=320 \mathrm{MPa})$, which in this case corresponded to $12 \mathrm{~N}$.

While the captured load was used directly in the contact analyses, the obtained images and the vertical displacements were post-processed to precisely define the size of the real contact area and the asperity deformations. In order to accurately determine the asperity deformations, the vertical displacement had to be measured with nanoscale resolution. However, when the deformations are controlled on this scale, any small deviations (i.e., deformations) arising from the bulk material beneath the asperities and deformations of test rig have to be considered and excluded from the asperity-deformation measurements. For this reason a polished reference sample with a roughness less than $\mathrm{Ra} 0.01 \mu \mathrm{m}$ was prepared and tested in exactly the same way as described in the previous section. We assumed that the measured deformations of a smooth sample were only a consequence of the bulk deformations of the specimen and the deformations of the test rig (Eq. (1)). The measured deformations of the reference specimen were subtracted from the deformations of the rough sample (Eq. (2)), measured under the same loads, to obtain the actual asperity deformations (Eq. (3)) [30].

$$
\begin{gathered}
d_{\text {reference }}=d_{\text {bulk }}+d_{\text {test rig }}, \\
d_{\text {sample }}=d_{\text {bulk }}+d_{\text {test rig }}+d_{\text {asperities }}, \\
d_{\text {asperities }}=d_{\text {sample }}-d_{\text {reference }} .
\end{gathered}
$$

During the experiments the images of the microcontacts between the specimen and the sapphire were captured with a CCD camera at a frame rate of $1 \mathrm{~Hz}$. When the specimen was moved into contact with the transparent window, micro-contacts between the asperities and the transparent window were observed. Moreover, because of the interference between the light reflected from the interface between the glass and air and the light reflected from the specimen, an optical fringe-pattern phenomenon was detected as well. If a threshold was applied to the obtained unprocessed image, fringes surrounding the actual contacts would produce false contacts, which would result in an overestimated contact area. To reduce this error and assess the actual contact area, each image was post-processed with the image-processing technique introduced by Krick et al. [29]. In Fig. 4 the contact between the specimen and the sapphire window is presented before and after the applied image-processing technique.
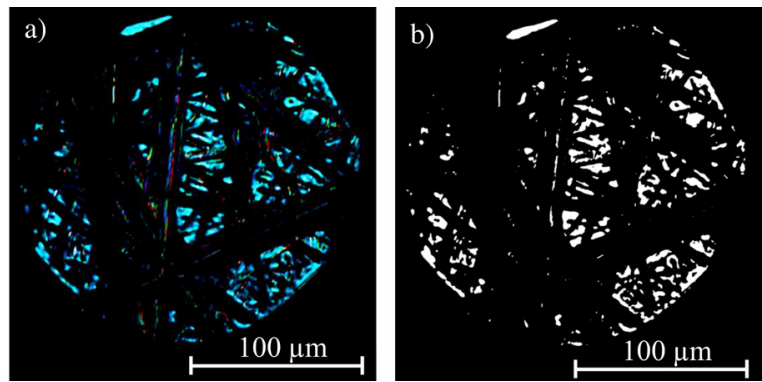

Fig. 4. Image of the contact between the deformable sample and the rigid sapphire window under a load of $10 \mathrm{~N}$; a) captured during the test and b) the resulting real contact area which was set by applying the image-processing technique [29] 
Several experiments were performed to obtain statistically relevant results. The variation among different samples at the same contact load in asperity deformations and real contact area was $8 \%$ and $18 \%$, respectively. However, for the consistency of surface features, data and analyses, results from only one sample are used throughout the analyses; namely, the one that is the closest to the mean values of analysed parameters.

\subsection{Theoretical Models for the Contact of Rough Surfaces}

In this work the results of the experiments were compared with theoretical calculations obtained using the two fundamental contact models, i.e., the GW model [1] considering only elastic deformations and the AF model [40] considering only plastic deformation, but modified with hardness criteria, as explained later on, namely the $\mathrm{AF}(H)$ model.

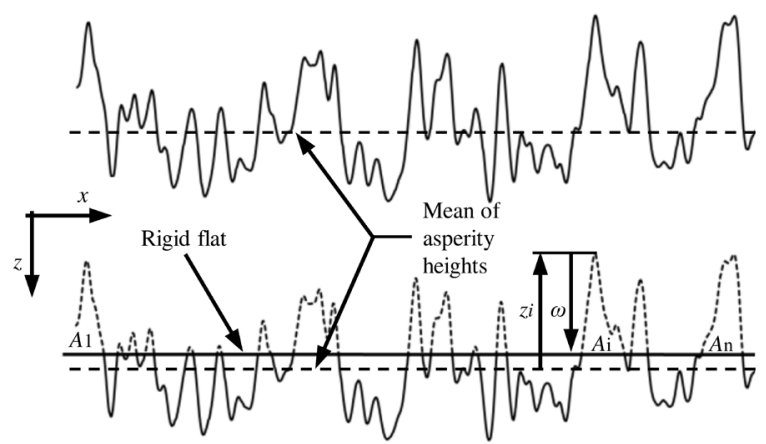

Fig. 5. Determination of the real contact area at certain degree of asperity deformations by slicing the 2-D surface profile according to the definition of the AF model

That is to say, in accordance with the definition of the AF model, the dependence between the real contact area $A r$ and the asperity deformations $\omega$ for the contact of a rigid and deformable flat was determined by truncation of the un-deformed surface (Fig. 5). Therefore, at any level of separation between the deformable and rigid flat $d=z-\omega$, the size of the real contact area was calculated by summing the intersections between two surfaces $\left(A_{i}\right)$ (Eq. (4)) [40].

$$
A r_{(A F)}(d)=A_{1}(d)+\ldots+A_{n}(d)=\sum_{i=1}^{n} A_{i}
$$

In the original work [40] authors did not determine any expression for calculating the load in fully plastic contacts. Therefore, in this work we introduce a "modified Abbott-Firestone" model, denoted as " $\mathrm{AF}(H)$ ", which assumes that for plastically deformed contacts the mean contact pressure remains constant and is equal to the material hardness $H$, as suggested by Bowden and Tabor [41]. The abbreviation $\operatorname{AF}(H)$ is used to explicitly show that the contact model for fully plastic deformation regime is not based only on AF model, which is usually misquoted by other researchers, but considers hardness criteria for deformation, as well.

Therefore, the contact loads for the fully plastic deformation regime can be calculated with the following equation:

$$
r(d)=A r_{(A F)}(d) \cdot H,
$$

where $H$ denotes hardness measured with microhardness tester.

To precisely assess the real contact area based on the AF model, the 3-D topography of the tested surface was measured prior to the actual testing. The topography was obtained with a 3-D optical interferometer (Contour GT-K0, Bruker, USA). A 10× magnification lens was used for the measurements, which resulted in the same lateral resolution in $x$ and $y$ directions $(\Delta x=\Delta y=0.334 \mu \mathrm{m})$ and a vertical resolution better than $0.1 \mathrm{~nm}$. Additionally, median filter [42] was used to eliminate any captured "artificial asperities" due to measurement errors, which could influence the theoretical results. Fig. 6 shows the surface topography of the tested sample measured with the interferometer. A quite good agreement between nominally flat surface and Gaussian distribution is also shown in Fig. 6, which is assumed in most of statistical contact models including GW model [43].

The second contact model used in this study was the GW model. It is probably the best-known statistical model for an elastic contact between a deformable engineering surface and an ideally flat, rigid counter surface. The contact as predicted by GW model [1] is schematically shown in Fig. 7. Based on the definition of the GW model, the real contact area and the contact load were calculated with the following equations:

$$
\begin{gathered}
A r_{(G W)}(d)=\pi N R A n \int_{d}^{\infty} \omega \phi(z) d z, \\
\operatorname{Pr}_{(G W)}(d)=\frac{4}{3} N E R^{1 / 2} A n \int_{d}^{\infty} \omega^{3 / 2} \phi(z) d z,
\end{gathered}
$$

where

$$
\frac{1}{E}=\frac{1-v_{1}^{2}}{E_{1}}+\frac{1-v_{2}^{2}}{E_{2}} .
$$

In Eqs. (6) and (7), $N$ and $R$ denote the asperity density and the average radius of the detected asperity tips, respectively; $A n$ is the nominal contact 

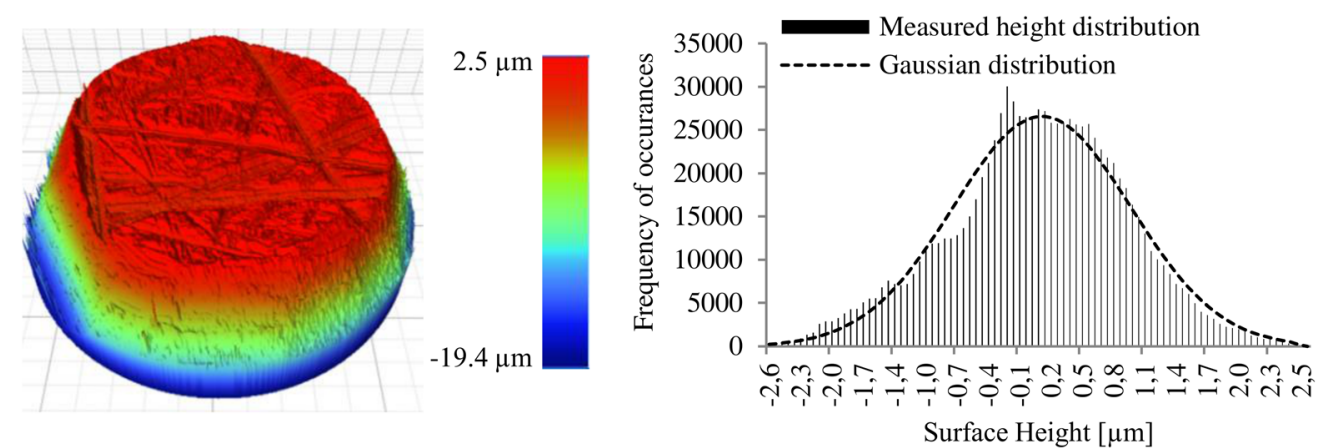

Fig. 6. Topography of the tested aluminium specimen, measured with the 3-D optical interferometer

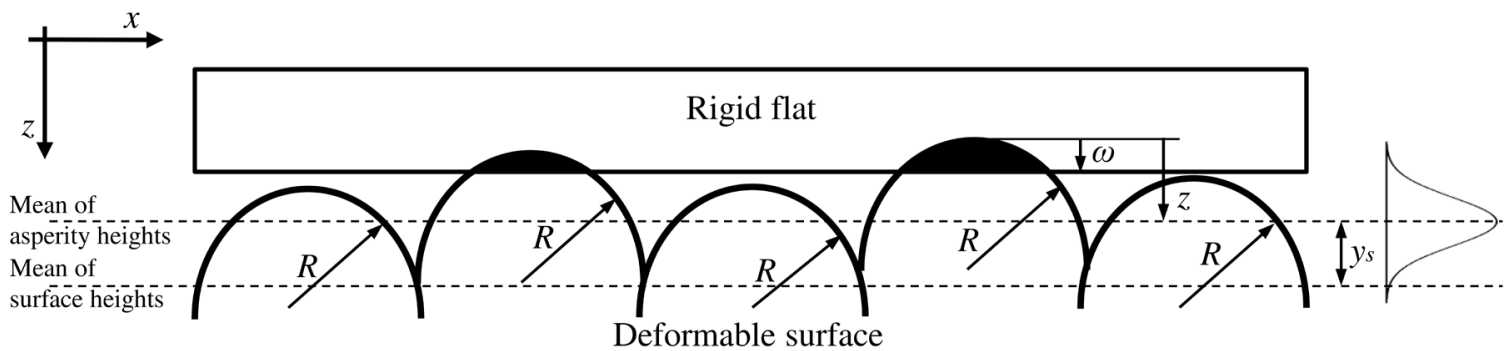

Fig. 7. Contact between the simplified deformable surface and rigid flat in accordance with the GW model [1]

area between two surfaces; $\phi(z)$ denotes the normal distribution function of the asperity heights; and $z$ represents the asperity height measured from the mean line of the summit heights. Eq. (8) presents the expression for calculating the equivalent Young's modulus $E$, where $E_{1}$ and $E_{2}$ are the Young's moduli and $v_{1}$ and $v_{2}$ are the Poisson's ratios of the contacting surfaces.

Table 2. Surface parameters of the analysed aluminium surface with a roughness $R a 0.6 \mu \mathrm{m}$ calculated using the $9 P P$ criterion

\begin{tabular}{lc}
\hline Asperity density $(N),\left[1 / \mathrm{m}^{2}\right]$ & $5.02 \mathrm{e}+10$ \\
\hline Average radius of detected asperity tips $(R),[\mathrm{mm}]$ & $1.70 \mathrm{e}-03$ \\
\hline The maximum asperity peak height $\left(Z_{\max }\right),[\mathrm{mm}]$ & $2.48 \mathrm{e}-03$ \\
\hline $\begin{array}{l}\text { Distance between the mean of asperity heights and } \\
\text { the mean of the surface heights }\left(y_{S}\right),[\mathrm{mm}]\end{array}$ & $0.83 \mathrm{e}-03$ \\
\hline Standard deviation of asperity heights $\left(\sigma_{\max }\right),[\mathrm{mm}]$ & $0.49 \mathrm{e}-03$ \\
\hline Standard deviation of surface heights $(\sigma),[\mathrm{mm}]$ & $0.85 \mathrm{e}-03$ \\
\hline
\end{tabular}

The material properties of the aluminium samples and the sapphire window were provided by the distributer and are specified in Table 1 . The surface parameters for the deformable specimen were determined from the same 3-D topography as presented in Fig. 6. The asperities on the surface needed to be determined arbitrarily. Therefore, the 9-point-peak (9PP) criterion [21] was adopted to detect and characterize the asperities. Besides $N$ and $R$, the maximum asperity peak height $\left(Z_{\max }\right)$, the distance between the mean of asperity heights and the mean of the surface heights $\left(y_{s}\right)$, the standard deviation of the asperity heights $\left(\sigma_{s}\right)$ and the standard deviation of the surface heights $(\sigma)$ were also calculated (Table 2) for a unified comparison of the experimental and theoretical results.

\section{RESULTS}

\subsection{Relationship between the Contact Load and the Asperity Deformations}

Fig. 8 shows the asperity deformations as a function of the contact load for the experimental measurements and the theoretical results obtained using the $\mathrm{GW}$ and $\mathrm{AF}(H)$ contact model.

The calculated asperity deformations for the same loads were the largest for the $\operatorname{AF}(H)$ model, where fully plastic deformations of the contacting asperities were assumed. The smallest deformations were calculated for the surfaces that were only deformed elastically, according to the GW model. It is clear that the experimental results are in better agreement with the GW model than with the $\mathrm{AF}(H)$ model over the whole region. Moreover, up to $2 \mathrm{~N}$, the deviations between the experimentally obtained data and the results predicted by the GW model are negligible (up 


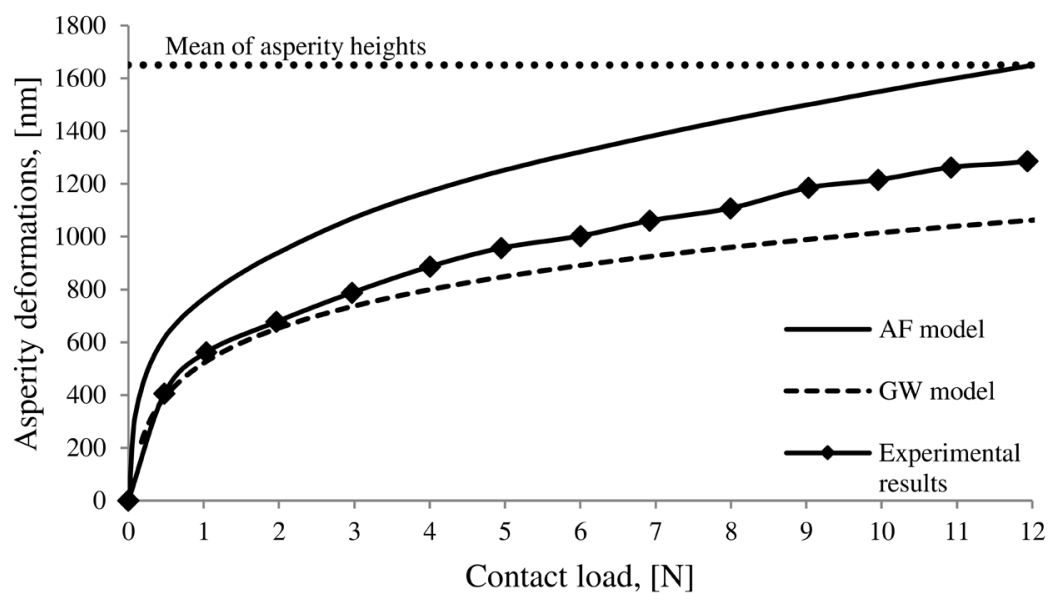

Fig. 8. Relationship between the contact load and asperity deformations, obtained experimentally and predicted using the $A F(H)$ and $G W$ models

to $3 \%$ at $2 \mathrm{~N}$ ). For loads above $2 \mathrm{~N}$ the experimental results start to deviate more significantly, also from the results of GW model and, in terms of the trend, i.e., slope, are more similar to the predictions of the $\mathrm{AF}(H)$ model. At a contact load of $12 \mathrm{~N}$ the measured asperity deformations are approximately $20 \%$ greater than those predicted by the GW model. In contrast, the largest discrepancy between the experimental data and the results of the $\operatorname{AF}(H)$ model is about $31 \%$, at $12 \mathrm{~N}$.

According to the GW model the deformation regime of a random surface can be predicted using the plasticity-index criterion [1], which is defined as:

$$
\Psi=(E / H) \cdot\left(\sigma_{s} / R\right)^{1 / 2} .
$$

By considering the material (Table 1) and topographic (Table 2) properties, the plasticity index $\Psi$ for the tested aluminium surface with a roughness $\mathrm{Ra}$ $0.6 \mu \mathrm{m}$ was 25 . Therefore, our surface should undergo plastic deformation and should, consequently, be in better agreement with the $\operatorname{AF}(H)$ model. However, as seen in Fig 8, the experimental results deviate significantly from these theoretical predictions.

Furthermore, while the $\operatorname{AF}(H)$ model predicts that the asperity deformations will match the mean value of the asperity heights at $12 \mathrm{~N}$, this level of deformation is not achieved within the loading range for the case of experimental measurements and the GW model.

\subsection{Relationship between the Asperity Deformations and $\mathrm{Ar} / \mathrm{An}$}

Fig. 9 shows $A r / A n$ as a function of the asperity deformations. It is clear that the theoretical results have a similar trend to the experimentally obtained data. Three regions can be seen in Fig. 9. When the asperity deformations are below $890 \mathrm{~nm}$ the measured $A r / A n$ values are larger than the theoretical predictions of the $\operatorname{AF}(H)$ model. The largest deviation between the experimental and theoretical results is at $670 \mathrm{~nm}$, where the experimentally measured $A r / A n$ value is $2.2 \%$ and the $A r / A n$ value predicted by the $\mathrm{AF}(H)$ model is $1.2 \%$. For the asperity deformations between $890 \mathrm{~nm}$ and $1,100 \mathrm{~nm}$ the differences between the experimental results and the predictions of the $\mathrm{AF}(H)$ model are negligible. With a further increase in the asperity deformations (i.e., $>1,100 \mathrm{~nm}$ ) the measured $A r / A n$ value is as much as $17 \%$ (at $1,280 \mathrm{~nm}$ ) smaller than the $A r / A n$ value predicted by the $\mathrm{AF}(H)$ model. On the other hand, the GW model predicts roughly up to 10-times smaller $A r / A n$ values than the experimental results across the deformation range.

Based on the dependency between $A r / A n$ and the asperity deformations we can claim that the results obtained using the $\operatorname{AF}(H)$ model are in better agreement with the experimental data than the predictions of the GW model, which is a contrast to the relationship between the contact load and the asperity deformations in Fig. 8.

\subsection{Relationship between the Contact Load and the Ar/An}

Fig 10a compares the experimentally obtained relationship between $A r / A n$ and the contact load with the results predicted by the $\mathrm{AF}(H)$ and GW models. In the case of the theoretical $\mathrm{GW}$ and $\mathrm{AF}(H)$ models the relationship between the contact load and $A r / A n$ is linear over the whole range of loads. It is clear that the experimental values lie in between the results obtained 


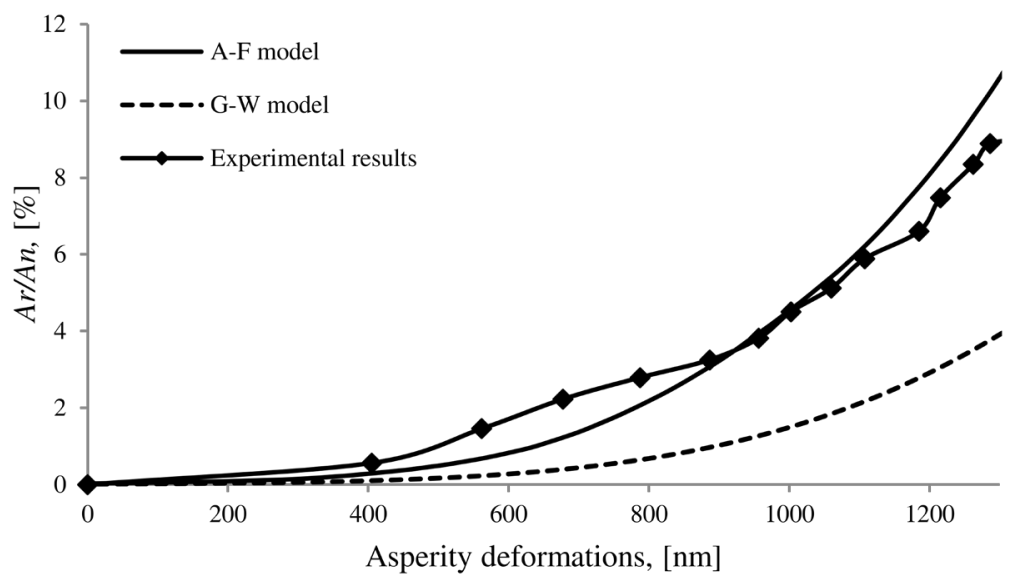

Fig. 9. Relationship between the asperity deformations and Ar/An, obtained experimentally and predicted by the $A F(H)$ and $G W$ models

(a)

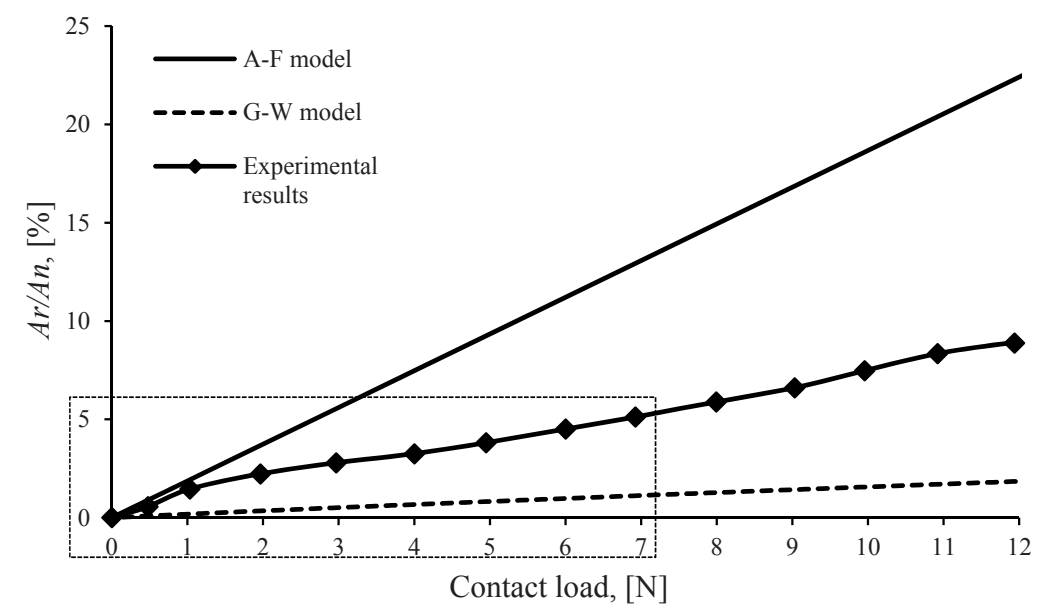

(b)

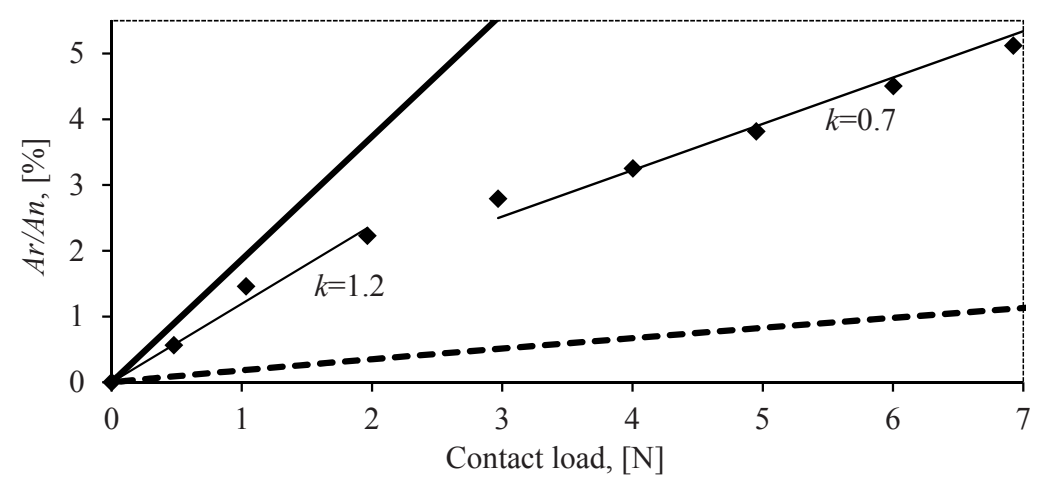

Fig. 10. a) The results for Ar/An depending on the contact load, obtained experimentally and predicted by the $A F(H)$ and $G W$ models; and $b)$ a detailed view from 10a with two separate regions for the relationship between the contact load and $\mathrm{Ar} / \mathrm{An}$

using the $\mathrm{GW}$ and $\mathrm{AF}(H)$ models over the whole loading region. The experimentally obtained $A r / A n$ is up to 5-times higher than that predicted using the $\mathrm{GW}$ model, while it is up to 2 or 3 times lower compared to the predictions of the $\operatorname{AF}(H)$ model, depending on the load. The deviations increase as the load increases.
The experimentally measured $A r / A n$ at the maximum contact load, i.e., $12 \mathrm{~N}$, is $9 \%$ (Fig. 10a).

However, a closer look (see Fig 10b) at the experimental results shows two separate regions for the experimental relationships between $A r / A n$ and the contact load. In both regions the relationship between 
$A r / A n$ and the contact load is fairly linear for the $\mathrm{GW}$ and $\operatorname{AF}(H)$ models. However, up to $2 \mathrm{~N}$, which corresponds to $600 \mathrm{~nm}$ of asperity deformation, the slope coefficient $(k)$ for the experimental data is 1.2 , and is in better agreement with the $\operatorname{AF}(H)$ model. In this region the GW model deviates significantly, i.e., a real contact area that is as much as a 10-times smaller. For the contact loads higher than $2 \mathrm{~N}$ (corresponding to an asperity deformation from $670 \mathrm{~nm}$ to 1,280 $\mathrm{nm})$, the slope coefficient for the experimental results is reduced to about 0.7 , and it becomes a little more like the GW model. This indicates that the actual real contact is not changing linearly with load, as the theoretical models would suggest, but it increases faster and becomes more plastic in nature at low loads, while at higher loads the increase in the real contact area is smaller and it is closer to elastic behaviour, which the GW model predicts.

\section{DISCUSSION}

This study looks at the contact between two flat surfaces. The results were obtained using a test rig that allows measurements of the asperity deformations and the real contact area with a submicron resolution, i.e., $20 \mathrm{~nm}$ and $700 \mathrm{~nm}$, respectively, and is controlled by the contact load with a resolution of $0.1 \mathrm{~N}$. Moreover, the test rig, together with the test specimens, is designed to monitor the full nominal contact area, thus including every possible asperity that can carry the load. This is very important in order to control all the asperities and their deformations together with the contact load from the moment when the first contact occurs, i.e., at a nanoscale resolution. In the multiasperity contact analyses of Azushima et al. [28], based on an optical method in a similar study to ours, the resolution of the test rig was lower and only a portion of the nominal contact area was captured during the experiment where the "unseen" asperity contacts can affect the results significantly. Therefore, we believe that a complete set of experimental contact parameters must be captured for accurate measurements, such as those developed in this test rig.

The analysed specimen with a roughness $\mathrm{Ra}$ $0.6 \mu \mathrm{m}$ was tested for the full range of possible engineering loads, i.e., from almost negligible up to a macro yield stress, which was calculated to initiate at a nominal contact load of $12 \mathrm{~N}$. Moreover, it should be noted that for a selected material and roughness, for the maximum contact load at yield $(12 \mathrm{~N})$, the asperities only deformed by $1,280 \mathrm{~nm}$, while their deformations at more typical engineering loads are much less, around several hundred $\mathrm{nm}$, indicating that the major part of the contact deformations is indeed on the nanoscale for the surfaces we used. This further shows the necessity for high precision, i.e., nanoscale, asperity deformation measurements.

Accordingly, at this experimental precision, we clearly show that the real contact area under such static loads can actually be very small. Only $9 \%$ of the nominal contact area was in contact at the maximum contact load at the yields stress, while for more typical loads around $0.5 \cdot Y$, i.e., $10 \mathrm{~N}$, this was $7.5 \%$. So far, this has not been experimentally shown at the precision we used or for the material and topographic properties similar to ours. In [28] the $\mathrm{Ar} / \mathrm{An}$ measured at the yield initiation was around $30 \%$; however, rougher $(\mathrm{Ra} 5.22 \mu \mathrm{m})$ and much softer $(Y=120 \mathrm{MPa})$ material was analysed in that study.

In this study the predictions of the fundamental $\mathrm{GW}$ and $\mathrm{AF}(H)$ contact models were also compared to the experimental results. There are two different aspects when comparing the theoretical and experimental results. Namely, when $A r / A n$ is analysed as a function of the asperity deformations, the relationship calculated with the predominantly plastic $\mathrm{AF}(H)$ model is very close to the experimental results, Fig. 9. Moreover, if we look at the calculated plasticity index (Eq. (9)), which for our tested surface predicts a fully plastic deformation at a value of $\Psi=25$, this relationship shows a very good agreement between this, very often used plasticity criterion [1], and actual contact behaviour in this work. Therefore, for the relationship between $A r / A n$ and the asperity deformations, the GW model was not appropriate.

The results at this point appear conventional and predictable, following the plasticity index criterion, which is indeed typically used. However, Fig. 8 clearly shows that the contact loads to achieve a certain micro-asperity deformation are much higher than those predicted by the $\mathrm{AF}(H)$ model. Accordingly, just the opposite of the above, when the relationships between the asperity deformations and the contact loads are analysed, the results of the GW model [1] for predominantly elastic deformations are in better agreement with the experimentally obtained relationship, Fig. 8, than the $\operatorname{AF}(H)$ model. This indicates that the contact loads predicted by the $\operatorname{AF}(H)$ model are underestimated for actual engineering contacts.

Accordingly, the two models are contradictory for the parameters analysed within the same contact. Moreover, even the very often used plasticity index that predicts the asperity deformation - irrespective of the real contact area and the load - shows a contradictory prediction, since when it is correlated 
with the real contact area it is in agreement with the $\mathrm{AF}(H)$ model and "properly predicts" the plastic deformation [44]; while when correlated with the load, the prediction is "wrong", since the experimental results show a similarity with the GW model, which predicts elastic behaviour.

These findings indicate that the actual contact behaviour within a multi-asperity contact cannot be accurately analysed with either of the two most commonly adopted theoretical models, i.e., the $\operatorname{AF}(H)$ or GW model, neither can it be properly anticipated using the plasticity index criterion.

The last observation, which can be made based on the obtained results, refers to the relationship between the contact load and $A r / A n$. While both theoretical contact models predict an almost linear relationship between the contact load and $A r / A n$, the experimental results show that the asperities have two different behaviours. At lower loads and, consequently, for smaller deformations, $A r / A n$ grows faster, Fig 10b. Therefore, the behaviour of $A r / A n$ with respect to the contact load is up to $0.2 \cdot Y$ (i.e. $2 \mathrm{~N}$ ) more similar to the predictions of the $\operatorname{AF}(H)$ model than GW, Fig 10b. However, at higher loads and deformations, the growth of $A r / A n$ slows down, so the trend becomes more like that of the GW model and thus more elastic. A similar trend was also observed by Jackson et al. [33] using a gold-coated rough ball. They suggested that the most plausible reason for such behaviour lies in strain hardening.

From our results it seems that the material properties change with load, probably due to strainhardening [33] and [45] or induced hydrostatic stresses [46]; however, the actual geometrical properties of the asperity [21] and [30] and interactions between neighbouring asperities [14] cannot be excluded from any influence since they also change simultaneously. This particular issue should be further analysed in detail to properly understand the mechanisms behind multi-asperity contact behaviour.

\section{CONCLUSIONS}

1. A test rig for in-situ experimental investigations of a multi-asperity contact under static loading conditions is presented. The apparatus allows measurements of the asperity deformations, the real contact area with a submicron resolution, 20 $\mathrm{nm}$ and $700 \mathrm{~nm}$, respectively, and the contact load with a resolution of $0.1 \mathrm{~N}$.

2. A careful specimen-preparation procedure and the optical specifications of the test rig make it possible to monitor the whole nominal contact area of the tested specimen, where each asperity can be detected when in contact. Therefore, no mechanical or topographic simplifications or assumptions are considered with the experimental approach presented in this work.

3. The comparison between the theoretical and experimental results indicates that comprehensive contact behaviour within a multi-asperity contact cannot be accurately predicted with only one of the two most commonly adopted theoretical models, e.g., $\mathrm{AF}(H)$ and $\mathrm{GW}$, since the predictions of the $\mathrm{AF}(H)$ model are in better agreement with the experimental results for the relationship between the asperity deformations and $A r / A n$, while the GW model better predicts the relationship between the asperity deformations and the contact load. Moreover, we experimentally showed that the actual contact behaviour cannot be properly anticipated using the plasticity-index criterion either.

4. The experimental results for the aluminium 6026 specimen with a roughness Ra $0.6 \mu \mathrm{m}$ show that at macro yield initiation only about $9 \%$ of the nominal contact area was in contact. Moreover, two distinctive regions for $A r / A n$ growth with respect to the contact load were observed from the experimental results. In contrast, the $\mathrm{GW}$ and $\mathrm{AF}(H)$ models predict a nearly linear relation. Therefore, an experimental analysis of the real contact area on the submicron level is crucial for any in-depth investigation of the actual contact behaviour.

A comparison between the experimental and theoretical results indicates discrepancies between the actual contact behaviour and the theoretical analysis. We believe that both the actual material and the topographic properties cause the deviations between theory and actual behaviour. Therefore, a further indepth investigation of the actual contact behaviour is necessary in order to properly understand the mechanisms in multi-asperity contacts.

\section{ACKNOWLEDGEMENTS}

The authors wish to acknowledge Slovenian Research Agency of the Republic of Slovenia for financial support.

\section{REFERENCES}

[1] Greenwood, J., Williamson, J. (1966). Contact of nominally flat surfaces. Proceedings of the Royal Society of London. Series 
A. Mathematical and Physical Sciences, vol. 295, no. 1442, p. 300-319, D0I:10.1098/rspa.1966.0242.

[2] Zhao, Y., Maietta, D.M., Chang, L. (1999). An asperity microcontact model incorporating the transition from elastic deformation to fully plastic flow. Journal of Tribology, vol. 122, no. 1, p. 86-93, D0I:10.1115/1.555332.

[3] Jackson, R.L., Green, I. (2006). A statistical model of elastoplastic asperity contact between rough surfaces. Tribology International, vol. 39, no. 9, p. 906-914, D0l:10.1016/j. triboint.2005.09.001.

[4] Kogut, L., Etsion, I. (2002). Elastic-plastic contact analysis of a sphere and a rigid flat. Journal of Applied Mechanics, vol. 69, no. 5, p. 657-662, Dol:10.1115/1.1490373.

[5] Liu, M., Proudhon, H. (2016). Finite element analysis of contact deformation regimes of an elastic-power plastic hardening sinusoidal asperity. Mechanics of Materials, vol. 103, p. 78-86, D0l:10.1016/j.mechmat.2016.08.015.

[6] Jeng, Y.-R., Peng, S.-R. (2005). Elastic-plastic contact behavior considering asperity interactions for surfaces with various height distributions. Journal of Tribology, vol. 128, no. 2, p. 245-251, DOl:10.1115/1.2162557.

[7] Rostami, A., Jackson, R.L. (2013). Predictions of the average surface separation and stiffness between contacting elastic and elastic-plastic sinusoidal surfaces. Proceedings of the Institution of Mechanical Engineers, Part J: Journal of Engineering Tribology, vol. 227, no. 12, p. 1376-1385, DOl:10.1177/1350650113495188.

[8] Zhao, B., Zhang, S., Qiu, Z. (2015). Analytical asperity interaction model and numerical model of multi-asperity contact for power hardening materials. Tribology International, vol. 92, p. 57-66, D0l:10.1016/j.triboint.2015.05.027.

[9] Beheshti, A., Khonsari, M. (2012). Asperity micro-contact models as applied to the deformation of rough line contact. Tribology International, vol. 52, p. 61-74, D0I:10.1016/j. triboint.2012.02.026.

[10] Buczkowski, R., Kleiber, M. (2009). Statistical models of rough surfaces for finite element 3D-contact analysis. Archives of Computational Methods in Engineering, vol. 16, no. 4, p. 399424, D0l:10.1007/s11831-009-9037-2.

[11] Xu, Y., Jackson, R.L. (2017). Statistical models of nearly complete elastic rough surface contact-comparison with numerical solutions. Tribology International, vol. 105, p. 274291, D0I:10.1016/j.triboint.2016.10.003.

[12] Majumdar, A., Bhushan, B. (1991). Fractal model of elasticplastic contact between rough surfaces. Journal of Tribology, vol. 113, no. 1, p. 1-11, D0l:10.1115/1.2920588.

[13] Ciavarella, M., Delfine, V., Demelio, G. (2006). A "re-vitalized" Greenwood and Williamson model of elastic contact between fractal surfaces. Journal of the Mechanics and Physics of Solids, vol. 54, no. 12, p. 2569-2591, D0l:10.1016/j. jmps.2006.05.006.

[14] Jackson, R.L., Streator, J.L. (2006). A multi-scale model for contact between rough surfaces. Wear, vol. 261, no. 11-12, p. 1337-1347, D0I:10.1016/j.wear.2006.03.015.

[15] Wilson, W.E., Angadi, S.V., Jackson, R.L. (2010). Surface separation and contact resistance considering sinusoidal elastic-plastic multi-scale rough surface contact. Wear, vol. 268, no. 1-2, p. 190-201, D0I:10.1016/j.wear.2009.07.012.
[16] Gao, Y.-F., Bower, A.F. (2006). Elastic-plastic contact of a rough surface with Weierstrass profile. Proceedings of the Royal Society A, vol. 462, no. 2065, p. 319-348, D0l:10.1098/ rspa.2005.1563.

[17] Belghith, S., Mezlini, S., BelhadjSalah, H., Ligier, J.-L. (2010). Modeling of contact between rough surfaces using homogenisation technique. Comptes Rendus Mécanique, vol. 338, no. 1, p. 48-61, D0l:10.1016/j.crme.2009.12.003.

[18] Jackson, R.L., Green, I. (2011). On the modeling of elastic contact between rough surfaces. Tribology Transactions, vol. 54, no. 2, p. 300-314, Dol:10.1080/10402004.2010.542277.

[19] Buchner, B., Buchner, M., Buchmayr, B. (2009). Determination of the real contact area for numerical simulation. Tribology International, vol. 42, no. 6, p. 897-901, D0I:10.1016/j triboint.2008.12.009.

[20] Pasaribu, H., Schipper, D. (2005). Application of a deterministic contact model to analyze the contact of a rough surface against a flat layered surface. Journal of Tribology, vol. 127, no. 2, p. 451-455, Dol:10.1115/1.1866163.

[21] Kalin, M., Pogačnik, A. (2013). Criteria and properties of the asperity peaks on 3D engineering surfaces. Wear, vol. 308, no. 1-2, p. 95-104, D0l:10.1016/j.wear.2013.09.010.

[22] Ovcharenko, A., Halperin, G., Etsion, I., Varenberg, M. (2006). A novel test rig for in situ and real time optical measurement of the contact area evolution during pre-sliding of a spherical contact. Tribology Letters, vol. 23, no. 1, p. 55-63, DOI:10.1007/s11249-006-9113-9.

[23] Kucharski, S., Starzynski, G. (2014). Study of contact of rough surfaces: Modeling and experiment. Wear, vol. 311, no. 1-2, p. 167-179, D0l:10.1016/j.wear.2014.01.009.

[24] Sick, J.-H., Ostermeyer, G.-P. (2007). In situ measurement of contact area in coated surfaces. Computer Methods and Experimental Measurements for Surface Effects and Contact Mechanics VIII, (De Hosson, J.T.M., Brebbia, C.A., Nishida, S.I., eds.) vol. 55, p. 259-270, D0I:10.2495/SECM070251.

[25] Bahrami, M., Yovanovich, M.M., Culham, J.R. (2005). Thermal contact resistance at low contact pressure: Effect of elastic deformation. International Journal of Heat and Mass Transfer, vol. 48, no. 16, p. 3284-3293, D0l:10.1016/j. ijheatmasstransfer.2005.02.033.

[26] Baltazar, A., Kim, J.Y., Rokhlin, S.I. (2006). Ultrasonic determination of real contact area of randomly rough surfaces in elastoplastic contact. Revista Mexicana de Física, vol. 52, no. 1, p. 37-47.

[27] Aymerich, F., Pau, M., Ginesu, F. (2003). Evaluation of nominal contact area and contact pressure distribution in a steelsteel interface by means of ultrasonic techniques. JSME International Journal Series C Mechanical Systems, Machine Elements and Manufacturing, vol. 46, no. 1, p. 297-305, DOI:10.1299/jsmec.46.297.

[28] Azushima, A., Kuba, S., Tani, S., Olsson, D.D. (2006). Direct observation of asperity deformation of specimens with random rough surfaces in upsetting and indentation processes. Wear, vol. 260, no. 3, p. 258-264, D0l:10.1016/j.wear.2005.04.022.

[29] Krick, B.A., Vail, J.R., Persson, B.N.J., Sawyer, W.G. (2012). Optical in situ micro tribometer for analysis of real contact area for contact mechanics, adhesion, and sliding experiments. 
Tribology Letters, vol. 45, no. 1, p. 185-194, D0l:10.1007/ s11249-011-9870-y.

[30] Pogačnik, A., Požar, T., Kalin, M., Možina, J. (2013). A homodyne quadrature laser interferometer for micro-asperity deformation analysis. Sensors, vol. 13, no. 1, p. 703-720, DOI:10.3390/s130100703.

[31] Matsuda, K., Hashimoto, D., Nakamura, K. (2016). Real contact area and friction property of rubber with twodimensional regular wavy surface. Tribology International, vol. 93, part B, p. 523-529, D0l:10.1016/j.triboint.2014.11.011.

[32] Ovcharenko, A., Halperin, G., Etsion, I. (2008). In situ and real-time optical investigation of junction growth in spherical elastic-plastic contact. Wear, vol. 264, no. 11-12, p. 10431050, D0l:10.1016/J.wear.2007.08.009.

[33] Jackson, R.L., Down, M.P., Liu, H., McBride, J.W. (2014). A comparison of the predictions of a multiscale model and optical real area of contact measurements. IEEE 60th $\mathrm{Holm}$ Conference on Electrical Contacts, p. 79-86, D0l:10.1109/ HOLM.2014.7031026.

[34] McBride, J.W., Cross, K.C. (2008). An experimental investigation of the contact area between a glass plane and both metallic and carbon-nano-tube electrical contacts. Proceedings of the 54th IEEE Holm Conference on Electrical Contacts, p. 325-331, DOI:10.1109/HOLM.2008.ECP.63.

[35] Nitta, I., Tsukiyama, Y., Tsukada, T., Terao, H. (2014). Measurement of real contact area on thermal print head using a laser microscope with a wide field of view. Tribology International, vol. 79, p. 162-173, Dol:10.1016/j. triboint.2014.06.005.

[36] Eguchi, M., Shibamiya, T., Yamamoto, T. (2009). Measurement of real contact area and analysis of stick/slip region. Tribology International, vol. 42, no. 11-12, p. 1781-1791, D0l:10.1016/j. triboint.2009.04.046.

[37] Ovcharenko, A., Halperin, G., Verberne, G., Etsion, I. (2007). In situ investigation of the contact area in elastic-plastic spherical contact during loading-unloading. Tribology Letters, vol. 25, no. 2, p. 153-160, Dol:10.1007/s11249-006-9164-y.

[38] Jamari, J., Schipper, D.J. (2007). Plastic deformation and contact area of an elastic-plastic contact of ellipsoid bodies after unloading. Tribology International, vol. 40, no. 8, p. 13111318, D0l:10.1016/j.triboint.2007.02.015.

[39] Brodnik Žugelj, B., Kalin, M. (2017). Submicron-scale experimental and theoretical analyses of multi-asperity contacts with different roughnesses. Tribology International, (communicated).

[40] Abbott, E., Firestone, F. (1933). Specifying surface quality: a method based on accurate measurement and comparison. Mechanical Engineering, vol. 55, p. 569-572.

[41] Bowden, F., Tabor, D. (1967). Friction and Lubrication, Methuen \& Co. Ltd., London.

[42] Rajni, R., Anutam, A. (2014). Image denoising techniques - An overview. International Journal of Computer Applications, vol. 86, no. 16, p. 13-17, Dol:10.5120/15069-3436.

[43] Lee, C.-H., Eriten, M., Polycarpou, A.A. (2010). Application of elastic-plastic static friction models to rough surfaces with asymmetric asperity distribution. Journal of Tribology, vol. 132, no. 3, p. 031602, D0l:10.1115/1.4001547.

[44] Jamari, J., Schipper, D.J. (2006). Experimental investigation of fully plastic contact of a sphere against a hard flat. Journal of Tribology, vol. 128, no. 2, p. 230-235, Dol:10.1115/1.2164470.

[45] Chatterjee, B., Sahoo, P. (2012). Effect of strain hardening on elastic-plastic contact of a deformable sphere against a rigid flat under full stick contact condition. Advances in Tribology, vol. 2012, p. 1-8, D0l:10.1155/2012/472794.

[46] Krithivasan, V., Jackson, R.L. (2007). An analysis of threedimensional elasto-plastic sinusoidal contact. Tribology Letters, vol. 27, no. 1, p. 31-43, D0l:10.1007/s11249-0079200-6. 